\title{
openheart Utility of balloon aortic valvuloplasty in the transcatheter aortic valve implantation era
}

\author{
Luke Dawson (1D , ${ }^{1,2}$ Alex Huang, ${ }^{1,3}$ Laura Selkrig, ${ }^{1}$ James A Shaw, ${ }^{1}$ \\ Dion Stub (D) , ${ }^{1,3}$ Antony Walton, ${ }^{1}$ Stephen J Duffy ${ }^{1,3}$
}

To cite: Dawson L, Huang A, Selkrig $\mathrm{L}$, et al. Utility of balloon aortic valvuloplasty in the transcatheter aortic valve implantation era. Open Heart 2020;7:e001208. doi:10.1136/ openhrt-2019-001208

Received 16 November 2019 Revised 23 January 2020 Accepted 10 February 2020

Check for updates

(c) Author(s) (or their employer(s)) 2020. Re-use permitted under CC BY-NC. No commercial re-use. See rights and permissions. Published by BMJ.

${ }^{1}$ Department of Cardiology, The Alfred Hospital, Melbourne, Victoria, Australia

${ }^{2}$ Department of Cardiology, The Royal Melbourne Hospital, Melbourne, Victoria, Australia ${ }^{3}$ Baker IDI Heart and Diabetes Institute, Melbourne, Victoria, Australia

Correspondence to Dr Stephen J Duffy; S.Duffy@ alfred.org.au

\section{ABSTRACT}

Background Balloon aortic valvuloplasty (BAV) has seen renewed interest since the advent of transcatheter aortic valve implantation (TAVI). The study aimed to characterise a contemporary BAV cohort and determinants of clinical outcomes.

Methods Patients undergoing BAV at a single tertiary centre were retrospectively reviewed over a 10-year period, and functional and mortality outcomes were reported with up to a 2-year follow-up.

Results 224 patients (aged 82.5 \pm 8.3 years; 48\% female) underwent BAV over the study period. Indications were either destination treatment $(39 \%)$ or bridge-to-valve replacement (61\%)—including bridge-to-decision (29\%), symptom relief while on the waitlist (27\%), and temporary contraindications to TAVI/aortic valve replacement (AVR) (5\%). The mean reduction of aortic mean pressure gradient was $38 \%$. Procedural mortality occurred in $0.5 \%$, stroke in $1.3 \%$, and major bleeding in $0.9 \%$. Twelvemonth mortality was $36 \%$ overall, and $26 \%$ and $50 \%$ in the bridging and destination groups, respectively. New York HeartAssociation (NYHA) class improved by $\geq 1$ at 30 days in 50\%. Among the bridge-to-TAVI/AVR group, $40 \%$ proceeded to TAVI/AVR within 12 months following BAV. In multivariate analysis, active malignancy at baseline (OR: $4.4,95 \% \mathrm{Cl}: 1.3$ to $15.1, \mathrm{p}=0.02$ ), smoking history (OR: $3.3,95 \%$ Cl: 1.3 to $7.9, p<0.01)$, LVEF $\leq 30 \%$ at baseline (OR: $3.2,95 \% \mathrm{Cl}: 1.3$ to $7.6, \mathrm{p}<0.01$ ), destination treatment (OR: $2.2,95 \%$ Cl: 1.0 to $4.9, p=0.04$ ) were all associated with 12-month mortality.

Conclusions BAV remains a useful procedure with relatively low rates of complications, however, 1-year mortality rates are high. Contemporary indications for BAV include a bridge to definitive valve replacement or destination treatment.

\section{INTRODUCTION}

Aortic stenosis (AS) is the most common valvular pathology in the elderly population and the preferred treatment in higher-risk populations has undergone major changes over the last 10 years with the development of transcatheter aortic valve implantation (TAVI) ${ }^{1-3}$ In this setting, there has been renewed interest in balloon aortic valvuloplasty (BAV), with increasing procedural

\section{Key questions}

What is already known about this subject?

- Balloon aortic valvuloplasty (BAV) has had a resurgence of interest since the advent of transcatheter aortic valve implantation. BAV may be used as a destination treatment for symptom improvement or as a bridge to transcatheter aortic valve implantation or surgical aortic valve replacement to stabilise patients while awaiting a definitive procedure.

What does this study add?

- This study concludes that BAV remains a safe, useful procedure for severe aortic stenosis, with low rates of complications, however, 1-year mortality rates are high. Predicting functional response following BAV has not previously been well studied, and symptomatic patients (New York Heart Association (NYHA) 3 or 4), female patients and obese patients were identified as the groups most likely to benefit from this procedure.

How might this impact on clinical practice?

- BAV is a useful clinical tool as destination therapy, where it provides effective symptomatic relief over 6-12 months, or as a bridge to definitive treatment where it can be used as a stratifying tool for clinical decision making on valve replacement, as a measure to address issues that pose temporary contraindication to TAVI/AVR and to offer symptomatic improvement to patients with prolonged waiting time, especially among the select groups identified in this study.

numbers both in the USA ${ }^{45}$ and other countries, ${ }^{67}$ which is a marked change from the previous steady decline since its early peak in the 1990s. ${ }^{8}$ Initially proposed as an alternative to surgical aortic valve replacement (AVR), ${ }^{9}$ BAV was shown to improve symptoms, although with significant complication rates and no long-term survival benefits. ${ }^{9-12}$ Traditional indications for BAV have been limited to poor surgical candidates and have included cardiogenic shock, ${ }^{813}$ preoperative optimisation $^{14}$ and symptomatic improvement with palliative intent. ${ }^{911} 1215$ Recently, a 
Table 1 Baseline characteristics

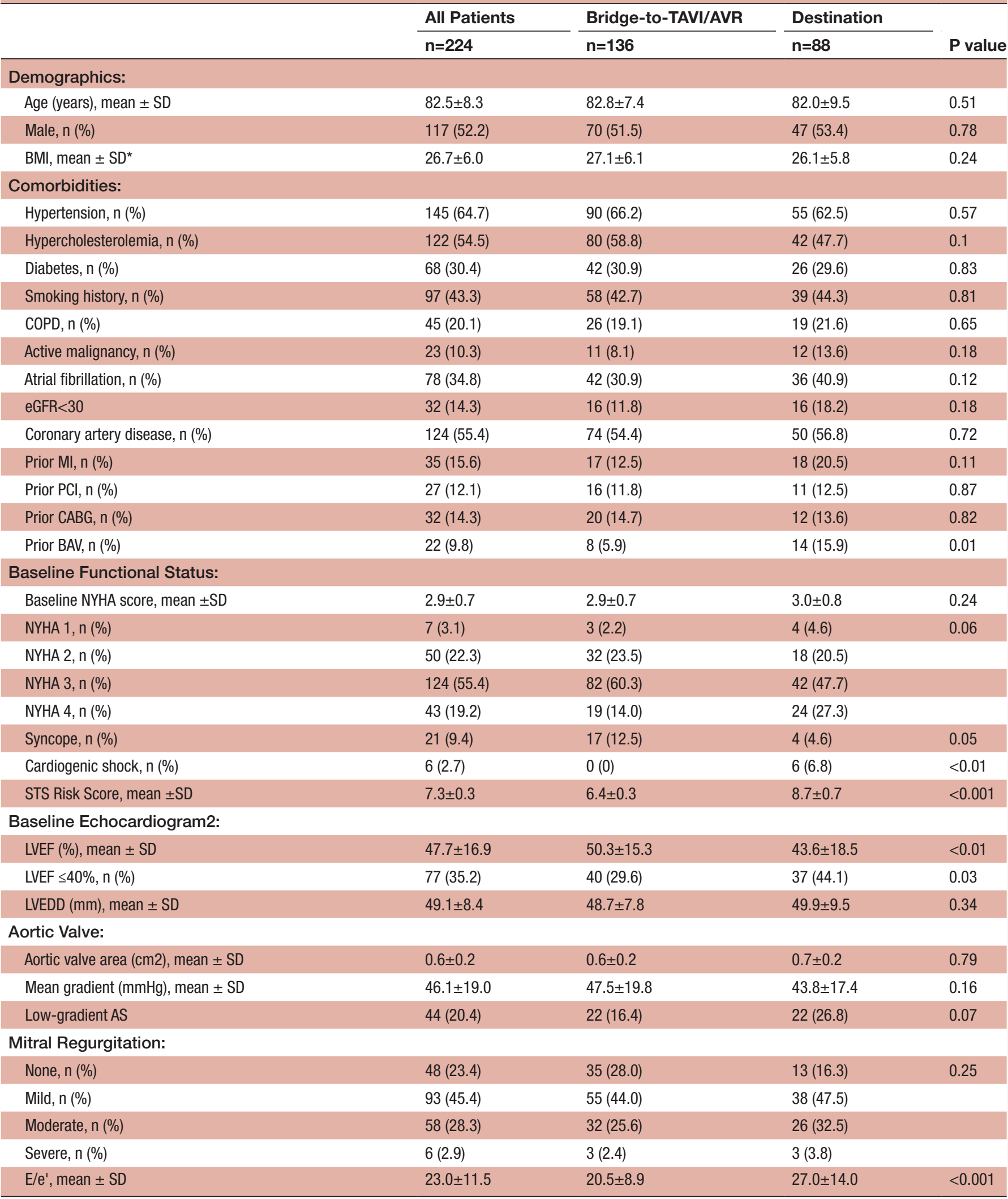

*207 BMI measures available.

†219 TTE measures prior to BAV available.

AS, aortic stenosis; BAV, balloon aortic valvuloplasty; BMI, body mass index; CABG, coronary artery bypass grafts; COPD, chronic obstruction pulmonary disease; eGFR, estimated glomerular filtration rate; LVEDD, left ventricular end-diastolic diameter; LVEF, left ventricular ejection fraction; MI, myocardial infarction; NYHA, New York Heart Association; PCI, percutaneous coronary intervention; STS, Society of Thoracic Surgery. 
more contemporary indication for BAV has included its utilisation as a bridge to either surgical or transcatheter AVR in patients who may have a temporary contraindication, ${ }^{16}$ who may derive uncertain benefit from TAVI or $\mathrm{AVR},{ }^{16-19}$ or when valve replacement is not immediately available. ${ }^{6} 716$ 19-23 Recent data suggest that emergency TAVI has a poor outcome, whereas BAV treatment may give time to prognosticate more accurately. ${ }^{24}$ Timely TAVI availability remains an important concern among this population given the high mortality among patients with severe AS and the potential for death among waitlisted patients, ${ }^{25}$ which may potentially be reduced with bridging BAV given its effect on initial improvement in survival. ${ }^{26}$ This study aimed to assess contemporary indications and outcomes for BAV, as well as to identify factors that predict functional, haemodynamic and mortality benefits to highlight select patient groups that may benefit most from BAV procedures in the TAVI era.

\section{MATERIALS AND METHODS}

Consecutive patients undergoing BAV for severe aortic stenosis from February 1, 2008 to September 1, 2017 at The Alfred Hospital, a large tertiary referrals centre in Melbourne, Australia, were retrospectively identified from the hospital cardiac database. Severe aortic stenosis was determined by transthoracic echocardiography prior to the procedure and was defined as either aortic valve area (AVA) $<1.0 \mathrm{~cm}^{2}$, Dimensionless Performance Index (DPI) $<0.25$ or mean gradient $\geq 40 \mathrm{~mm} \mathrm{Hg}$ in the setting of normal left ventricular (LV) function on transthoracic echocardiography. Severe aortic stenosis in the setting of abnormal LV function was determined by an increase in mean gradient to $\geq 40 \mathrm{~mm} \mathrm{Hg}$ with dobutamine stress transthoracic echocardiogram (TTE) or by Heart Team assessment. Patients with mixed aortic valve disease (moderate or severe aortic regurgitation in addition to AS) are not assessed for BAV at our centre and are not included in the study. The project was approved by the Alfred Hospital Ethics Committee.

Demographic data were obtained through a detailed review of the hospital electronic medical records to determine patient demographics, comorbidities, cardiovascular risk factors, cardiac symptoms and presence of cardiogenic shock prior to the procedure. Functional status was determined from medical records by trained medical personnel by applying New York Heart Association (NYHA) criteria to the nearest clinical encounter describing functional ability within 30 days preceding $\mathrm{BAV}$ procedure. Indication for BAV was determined by clinician intent at the time of BAV procedure (eg, bridgeto-TAVI/AVR) and patients remained in their intentionto-treat grouping irrespective of the eventual outcome (ie, bridge-to-TAVI and bridge-to-AVR patients did not necessarily receive a valve replacement procedure). Throughout the study period, over 300 TAVI procedures were performed at our institution, with 70 TAVIs performed in 2016 and $>90$ procedures projected for 2017.

BAV procedures were performed by three individual operators (SD, AW, DS) using standard techniques via the retrograde femoral approach in all patients. Peak and mean gradient measures were confirmed with simultaneous catheter gradient measures. NuMED NuCLEUS and Pyramed Cristal aortic balloons are used at our institution. Sheath size, balloon size, preprocedural and postprocedural haemodynamics, immediate complications and in-hospital mortality were determined from the electronic procedural and clinical records. In-hospital complications, including myocardial infarction and stroke, were defined according to the Academic Research Consortium definitions. ${ }^{27}$ Significant bleeding was defined as Bleeding Academic Research Consortium Classification Type 3 or $5 .{ }^{28}$ Acute kidney injury (AKI) following $\mathrm{BAV}$ was defined as increase in serum creatinine $\geq 26.5$ $\mu \mathrm{mol} / \mathrm{L}$ within 48 hours or increase in creatinine to $\geq 1.5$ times baseline taken within the prior 7 days according to Kidney Disease: Improving Global Outcomes (KDIGO) criteria. $^{29}$

Patient outcomes were assessed at 30 days, 6 months and 12 months post procedure. Clinic notes and subsequent admissions were used to determine NYHA functional status and hospital readmission rates. TTE reports were reviewed to determine LV ejection fraction (LVEF), AVA, LV end-diastolic diameter (LVEDD), E/e' and severity of mitral regurgitation (trivial, mild, moderate or severe). Patient mortality was assessed to at least 12 months using clinical records and telephone follow-up, with days from procedure to death used to generate Kaplan-Meier survival curves. If a patient received TAVI or AVR during the 12 months post BAV, then they were no longer included in functional or TTE response after

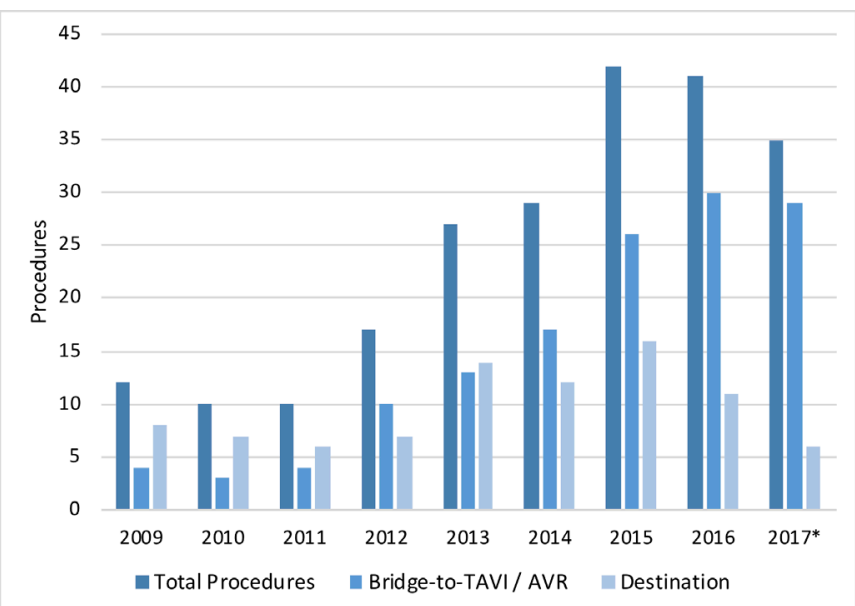

Figure 1 Procedural numbers by indication - procedural numbers at a single institution over a 9-year period. *2017 data only includes up to September 2017 (with projected numbers for the 2017 calendar year being 45 bridge-toTAVI/AVR procedures and 8 destination procedures). AVR, aortic valve replacement; TAVI, transcatheter aortic valve implantation. 


\begin{tabular}{cc}
\hline Table 2 Indications for BAV & \\
\hline & N=224 \\
\hline Bridge-to-TAVI/SAVR, $\mathrm{n}(\%)$ & $136(60.7)$ \\
\hline Bridge-to-decision & $64(28.6)$ \\
BAV response used in decision* & $25(11.1)$ \\
\hline Prolonged procedural waiting time & $60(26.8)$ \\
\hline Temporary contraindication to TAVI/AVR & $12(5.4)$ \\
\hline Destination treatment, $\mathrm{n}(\%)$ & $88(39.3)$ \\
\hline Preoperative optimisation† & $10(4.5)$ \\
\hline Cardiogenic shock & $8(3.6)$ \\
Symptom improvement & $70(31.3)$ \\
\hline
\end{tabular}

${ }^{*}$ Response to BAV (improvement in LV function or functional status after BAV) used to determine likely response to TAVI/SAVR. †BAV performed to reduce perioperative risks in patients with severe AS undergoing non-cardiac surgery. AS, aortic stenosis; AVR, aortic valve replacement; BAV, balloon aortic valvuloplasty; SAVR, surgical aortic valve replacement; TAVI, transcatheter aortic valve implantation.

this point, however, continued to be followed for postprocedural mortality to at least 12 months. If a patient received a repeat $\mathrm{BAV}$ within 12 months, then the patient was censored from follow-up at the time of repeat BAV and included as a new procedure.

Statistical analysis was performed using Stata V.14.2 for Macintosh. All categorical variables are presented as frequencies and percentages and continuous variables are presented as mean \pm SD. Comparisons between groups were performed with the $\chi^{2}$ test for categorical variables and Student $t$ test for continuous variables. Multivariate analysis was performed using logistic regression to determine independent predictors of 12 month mortality, functional response (defined by NYHA score improvement $\geq 1$ at 30 days) and haemodynamic response (defined by improvement in LVEF by $\geq 10 \%$ at 30 days). Variables with a $\mathrm{p}<0.10$ in univariate analysis were considered for inclusion in multivariate analysis. The cumulative probability of survival was estimated by Kaplan-Meier curves, with survival between indication groups compared using the log-rank test. All calculated $\mathrm{p}$ values were two-sided and $p$ values of $<0.05$ were considered statistically significant.

\section{RESULTS}

Two hundred and twenty-four patients were included in the cohort; the mean age was $82.5 \pm 8.3$ years, and $47.8 \%$ were female (table 1). Procedural numbers increased across the study period, mainly driven by an increase in the number of bridge-to-TAVI/AVR procedures performed, in line with an increase in TAVI procedural numbers across the same period (figure 1).

Baseline clinical characteristics were similar in the bridge-to-TAVI/AVR and destination indication groups, however, there was a higher rate of prior BAV in the destination group ( $15.9 \%$ vs $5.9 \%, \mathrm{p}=0.01)$. Syncope was more common among the bridge-to-TAVI/AVR group $(\mathrm{p}=0.05)$, whereas cardiogenic shock was more common in the destination group $(\mathrm{p}<0.01)$. Mean Society of Thoracic Surgery (STS) Risk Score was lower in the bridge-toTAVI/AVR group $(6.4 \pm 0.3 \%$ vs $8.7 \pm 0.7 \%$, $\mathrm{p}<0.001)$. Baseline echocardiographic data are presented in table 1 , with the destination group having lower mean LVEF $(43.6 \pm 18.5 \%$ vs $50.3 \pm 18.5 \%, \mathrm{p}<0.01)$, and higher mean $\mathrm{E} / \mathrm{e}$ ' ratios $(27.0 \pm 14.0$ vs $20.5 \pm 8.9, \mathrm{p}<0.001)$ compared with the bridge-to-TAVI/AVR group. Indications for BAV are presented in table 2.

Procedural characteristics are presented in table 3. One hundred and nine procedures $(48.7 \%)$ were performed electively, with 84 of these $(37.5 \%)$ performed at the time of TAVI workup with coronary and peripheral angiography. The mean gradient reduction was $15.9 \pm 9.5 \mathrm{~mm}$ $\mathrm{Hg}$, with 144 procedures $(66.4 \%)$ reducing the gradient by $\geq 30 \%$. Balloon size used was $18 \mathrm{~mm}$ in $56.6 \%, 20 \mathrm{~mm}$ in $23.7 \%$ and $22 \mathrm{~mm}$ in $11.4 \%$, with other sizes being less common.

Major vascular complications occurred in $3.1 \%$, stroke in $1.3 \%$, major bleeding in $0.9 \%$ and in-hospital mortality was $2.2 \%$. Procedural mortality occurred in only one patient due to acute aortic regurgitation, while undergoing urgent inpatient BAV for intractable NYHA class 4 symptoms in the setting of low-flow AS. An $18 \mathrm{~mm}$ balloon size was used with single inflation, and no clear precipitant for acute aortic regurgitation was identified. There were no significant differences in procedural characteristics or in-hospital complications between the bridge-toTAVI/AVR cohort and the destination cohort.

For destination and bridge-to-TAVI/AVR groups, mortality was $50.0 \%$ and $26.3 \%$ at 12 months $(p<0.01)$, respectively (table 4 ). Eighty-six patients had a repeat aortic procedure: 18 patients had repeat BAV (median time to BAV 357 days), 56 patients proceeded to TAVI (median time to TAVI 135 days) and 12 patients proceeded to AVR (median time to AVR 128 days). Of the 136 patients receiving BAV for bridge-to-TAVI/AVR, only 54 patients $(39.7 \%)$ proceeded to TAVI or AVR within 12 months following BAV, with many patients $(60.3 \%)$ not proceeding to TAVI due to either death or Heart Team decision that they were unlikely to benefit. Twelve-month mortality was $7.0 \%$ among those proceeding to TAVI or AVR.

NYHA functional response to $\mathrm{BAV}$ is presented in figure 2. Of the cohort, $74.6 \%$ were NYHA grade 3 or 4 at baseline, compared with $34.8 \%$ at 30 days, $55.4 \%$ at 6 months, and $68.9 \%$ at 12 months $(\mathrm{p}<0.001)$. Multivariate analysis demonstrated NYHA 4 at baseline, NYHA 3 at baseline, body mass index (BMI) $\geq 30 \mathrm{~kg} / \mathrm{m}^{2}$, female gender, and bridge-to-TAVI/AVR as the indication for the $\mathrm{BAV}$, which were all associated with NYHA grade improvement of $\geq 1$ at 30 days post BAV (table 5).

The mean aortic valve area was $0.65 \pm 0.2 \mathrm{~cm}^{2}$ at baseline, $0.79 \pm 0.3 \mathrm{~cm}^{2}$ at 30 days, $0.73 \pm 0.2 \mathrm{~cm}^{2}$ at 6 months and $0.66 \pm 0.2 \mathrm{~cm}^{2}$ at 12 months. The mean aortic mean gradient was $46.1 \pm 19.0 \mathrm{~mm} \mathrm{Hg}$ at baseline (pre-BAV), $40.4 \pm 0.2 \mathrm{~mm} \mathrm{Hg}$ at 30 days $(\mathrm{p}<0.001$ compared with 
Table 3 Procedural characteristics and complications

\begin{tabular}{|c|c|c|c|c|}
\hline & All patients & $\begin{array}{l}\text { Bridge-to-TAVI/ } \\
\text { AVR }\end{array}$ & Destination & \\
\hline & $\mathrm{n}=224$ & $n=136$ & $n=88$ & $P$ value \\
\hline \multicolumn{5}{|l|}{ Procedural characteristics } \\
\hline Elective procedure, n(\%) & $109(48.7)$ & $65(47.8)$ & $44(50.0)$ & 0.75 \\
\hline During TAVI workup angiogram, $\mathrm{n}(\%)$ & $84(37.5)$ & $84(61.8)$ & $0(0)$ & $<0.001$ \\
\hline Balloon size, mean \pm SD & $19 \pm 2$ & $19 \pm 2$ & $19 \pm 2$ & 0.11 \\
\hline Sheath size, mean $\pm S D$ & $10 \pm 2$ & $10 \pm 2$ & $10 \pm 1$ & 0.17 \\
\hline \multicolumn{5}{|l|}{ Inflations, n (\%) } \\
\hline 1 & $73(79.7)$ & $107(81.7)$ & $66(76.7)$ & 0.47 \\
\hline 2 & $36(16.6)$ & $18(13.7)$ & $18(21.0)$ & \\
\hline$\geq 3$ & $8(3.6)$ & $6(4.6)$ & $2(2.3)$ & \\
\hline LVEDP $(\mathrm{mm} \mathrm{Hg})$, mean \pm SD & $18.0 \pm 20.2$ & $18.6 \pm 22.9$ & $16.7 \pm 11.4$ & 0.67 \\
\hline \multicolumn{5}{|l|}{ Mean gradient $(\mathrm{mm} \mathrm{Hg})$} \\
\hline Pre-BAV, mean $\pm S D$ & $41.6 \pm 18.1$ & $42.2 \pm 18.1$ & $40.8 \pm 18.0$ & 0.57 \\
\hline Post-BAV, mean $\pm S D$ & $26.2 \pm 13.4$ & $26.2 \pm 13.3$ & $26.1 \pm 13.5$ & 0.97 \\
\hline Reduction, mean $\pm S D$ & $15.9 \pm 9.5$ & $16.5 \pm 9.4$ & $14.9 \pm 9.7$ & 0.23 \\
\hline Reduced by $\geq 30 \%$, n (\%) & $144(66.4)$ & $93(69.9)$ & $51(60.7)$ & 0.16 \\
\hline \multicolumn{5}{|l|}{ In-hospital complications } \\
\hline Any complication, $\mathrm{n}(\%)$ & $18(8.0)$ & $11(8.1)$ & $7(8.0)$ & 0.59 \\
\hline Vascular complications, n (\%) & $7(3.1)$ & $5(3.7)$ & $2(2.3)$ & 0.56 \\
\hline Myocardial infarction, $\mathrm{n}(\%)$ & $1(0.5)$ & $1(0.7)$ & $0(0)$ & 0.42 \\
\hline Major bleeding (BARC $\geq 3$ ), n (\%) & $2(0.9)$ & $1(0.7)$ & $1(1.1)$ & 0.76 \\
\hline Acute kidney injury, n (\%) & $2(0.9)$ & $1(0.7)$ & $1(1.1)$ & 0.76 \\
\hline Stroke, n (\%) & $3(1.3)$ & $1(0.7)$ & $2(2.3)$ & 0.33 \\
\hline Acute aortic regurgitation, $\mathrm{n}(\%)$ & $1(0.5)$ & $0(0)$ & $1(1.1)$ & 0.21 \\
\hline Pacemaker insertion, $\mathrm{n}(\%)$ & $2(0.9)$ & $2(1.5)$ & $0(0)$ & 0.25 \\
\hline In-hospital mortality n (\%) & $5(2.2)$ & $1(0.7)$ & $4(4.6)$ & 0.06 \\
\hline Procedural mortality, n (\%) & $1(0.5)$ & $0(0)$ & $1(1.1)$ & 0.21 \\
\hline
\end{tabular}

LVEDP and mean gradient measured by catheter during procedure. Major vascular complications include false aneurysm, dissection or complication requiring surgery or percutaneous intervention (simple haematoma not included in major vascular complications unless meeting criteria for major bleeding, that is, BARC $\geq 3$ ).

AVR, aortic valve replacement; BARC, Bleeding Academic Research Consortium; BAV, balloon aortic valvuloplasty; LVEDP, left ventricular end-diastolic pressure; TAVI, transcatheter aortic valve implantation.

baseline), $43.8 \pm 15.4 \mathrm{~mm} \mathrm{Hg}$ at 6 months $(\mathrm{p}=0.65)$ and $47.8 \pm 24.0 \mathrm{~mm} \mathrm{Hg}$ at 12 months $(\mathrm{p}=0.19)$. The mean LVEF, E/e' and LVEDD measures were not different at 30 days, 6 months or 12 months following BAV.

LVEF improved by $\geq 10 \%$ in the destination treatment group in $15.6 \%$ at 30 days and $7.1 \%$ at 6 months, and in the bridge-to-TAVI/AVR group in $15.7 \%$ at 30 days and $21.2 \%$ at 6 months (table 4 ). Among patients with LVEF $\leq 40 \%$ at baseline, $24.3 \%$ had improvement by $\geq 10 \%$ at 30 days and $47.8 \%$ had improvement by $\geq 10 \%$ at 6 months. In multivariate analysis, LVEF $\leq 40 \%$ at baseline was the only variable associated with improvement in LVEF by $\geq 10 \%$ at 6 months (table 5 ).

Forty-four patients $(20.4 \%)$ had low-gradient AS, of which 22 had a BAV indication of bridge-to-TAVI/AVR. Of those with available TTE data, 4 of 21 patients (19.1\%) demonstrated improvement in LVEF $\geq 10 \%$ at 30 days, whereas 7 of 15 patients $(46.7 \%)$ demonstrated improvement in LVEF $\geq 10 \%$ at 6 months. BAV was performed in 7 of the 22 bridge-to-TAVI/AVR patients with the intent of assessing for improvement in LVEF, and of these, 2 patients proceeded to TAVI, whereas 1 patient proceeded to AVR following a good response to BAV.

Multivariate analysis identified active malignancy at baseline, smoking history, LVEF $\leq 30 \%$ at baseline, nonelective procedure and destination treatment as the indication, which were all associated with 12-month mortality following BAV (table 5). Kaplan-Meier survival estimates are presented by indication in figure 3 , demonstrating a significant difference in survival between destination and bridge-to-TAVI/AVR groups $(\mathrm{p}<0.01)$ to 24 months. Degree of reduction in catheter-measured mean pressure 


\begin{tabular}{|c|c|c|c|}
\hline & Bridge-to-TAVI/AVR & Destination & $P$ value \\
\hline 30 days, $\mathrm{n}(\%)$ & $n=130$ & $\mathrm{n}=83$ & \\
\hline NYHA improved $\geq 1^{*}$ & $71(60.2)$ & $32(46.4)$ & 0.07 \\
\hline LVEF improved $\geq 10 \% \dagger$ & $8(15.7)$ & 7 (15.6) & 0.99 \\
\hline Readmission & $22(16.8)$ & $9(10.8)$ & 0.23 \\
\hline Mortality & $2(1.5)$ & $9(10.8)$ & $<0.01$ \\
\hline 6 months, n (\%) & $\mathrm{n}=111$ & $\mathrm{n}=77$ & \\
\hline NYHA improved $\geq 1^{*}$ & $39(38.2)$ & $21(32.8)$ & 0.48 \\
\hline LVEF improved $\geq 10 \% \dagger$ & $11(21.2)$ & $1(7.1)$ & 0.23 \\
\hline Readmission & $41(36.3)$ & $31(38.8)$ & 0.73 \\
\hline Mortality & $17(15.3)$ & $25(32.5)$ & $<0.01$ \\
\hline 12 months, $\mathrm{n}(\%)$ & $\mathrm{n}=95$ & $\mathrm{n}=70$ & \\
\hline NYHA improved $\geq 1^{*}$ & $18(32.1)$ & $9(15.3)$ & 0.03 \\
\hline Readmission & $49(48.0)$ & $31(43.1)$ & 0.52 \\
\hline Mortality & $25(26.3)$ & $35(50.0)$ & $<0.01$ \\
\hline
\end{tabular}

*Functional data available for 187 patients at 30 days, 166 patients at 6 months and 115 patients at 12 months. Note functional scoring not performed for patients following a repeat aortic procedure (including TAVI, SAVR).

†TTE data for LVEF available for 96 patients at 30 days, and 66 patients at 6 months; 12 -month data not included due to limited number of available TTEs.

AVR, aortic valve replacement; BAV, balloon aortic valvuloplasty; LVEF, left ventricular ejection fraction; NYHA, New York Heart Association; SAVR, surgical aortic valve implantation; TAVI, transcatheter aortic valve implantation; TTE, transthoracic echocardiogram.

gradient immediately post BAV was associated with reduced 12 month mortality in univariate but not multivariate analysis, but was not associated with improvements in NYHA score. At 12 months, $76 \%$ had an NYHA score $\geq 2$, consistent with severe symptomatic AS (figure 2).

\section{DISCUSSION}

This study focuses on the current indications, use and outcomes of BAV in the contemporary setting, where TAVI is commonly used. Among our cohort, $57 \%$ of procedures were performed as a bridge-to-TAVI/AVR (and is increasing year by year), demonstrating that this is increasingly becoming accepted as an indication for BAV. This is in line with current American College of Cardiology/American Heart Association guidelines stating BAV may be considered as a bridge to AVR or TAVI in patients with severe symptomatic AS (Class 2B; Level of Evidence C)..$^{30}{ }^{31}$ Of note, in our cohort, BAV was used as a bridge-to-decision in $29 \%$ of the cases. Although procedural mortality for BAV is low $(<1 \%)$ and there is significant symptomatic benefit following BAV, by 12 months there is a recurrence of severe, symptomatic AS in $76 \%$ of patients. Thus, definitive therapy such as TAVI or AVR should be offered in the first 6 months following BAV, where appropriate.

Frequently, given the age, frailty and multiple comorbidities of patients with severe aortic stenosis, potential benefits of TAVI or AVR may be uncertain, and with this uncertainty, it may be difficult to justify the procedural risks and costs associated with TAVI/AVR. BAV has utility in this context for two reasons. First, as proposed by previous studies, ${ }^{12-14}$ patient response to BAV can be used to delineate the contribution of aortic stenosis to patients' symptoms and functional impairment, relative to other limiting comorbidities. It provides the clinical prediction of likely functional and haemodynamic response to a valve replacement and thus allows better patient selection. This indication accounted for $11 \%$ of our cohort. Second, patients with severe aortic stenosis often have complex medical histories that require further

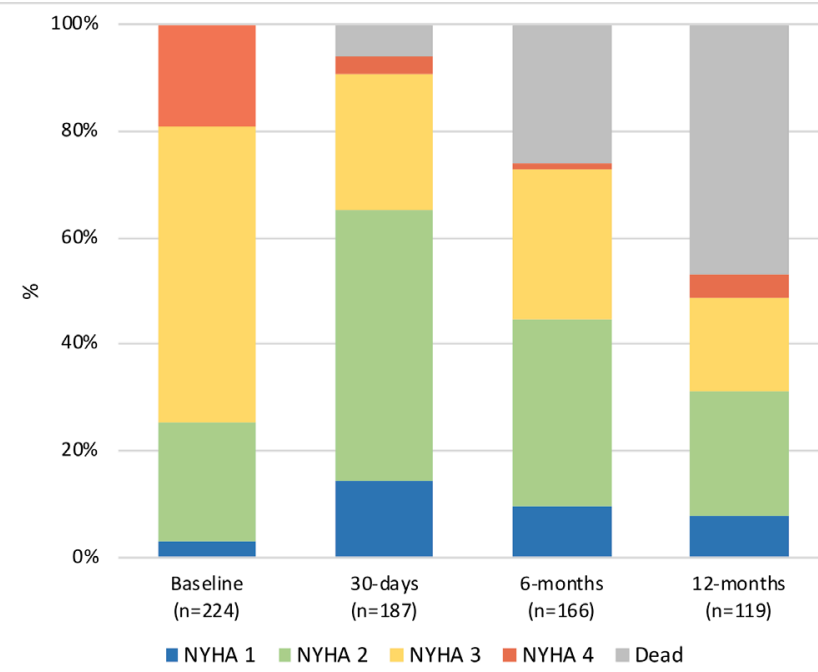

Figure 2 Functional response to BAV - functional response to BAV assessed using NYHA scores to classify symptoms. BAV, balloon aortic valvuloplasty; NYHA, New York Heart Association. 
Table 5 Multivariate predictors of response to BAV

Multivariate predictors of improvement of $\geq 10 \%$ in LVEF at 6 months post BAV

\begin{tabular}{|c|c|c|c|}
\hline & OR & $95 \% \mathrm{Cl}$ & $P$ value \\
\hline LVEF $\leq 40 \%$ at baseline & 26.8 & $3.0-238.2$ & $<0.01$ \\
\hline \multicolumn{4}{|c|}{$\begin{array}{l}\text { Multivariate predictors of NYHA score improvement of } \geq \\
\text { at } 30 \text { days post BAV }\end{array}$} \\
\hline & OR & $95 \% \mathrm{Cl}$ & $P$ value \\
\hline NYHA 4 at baseline & 14.2 & $4.4-45.1$ & $<0.001$ \\
\hline NYHA 3 at baseline & 4.8 & $2.0-11.4$ & $<0.001$ \\
\hline $\mathrm{BMI} \geq 30 \mathrm{~kg} / \mathrm{m}^{2}$ & 3.0 & $1.3-6.8$ & $<0.01$ \\
\hline Female & 2.1 & $1.0-4.3$ & 0.04 \\
\hline $\begin{array}{l}\text { Indication: bridge-to- } \\
\text { TAVI/SAVR }\end{array}$ & 1.9 & $0.9-3.9$ & 0.08 \\
\hline \multicolumn{4}{|c|}{ Multivariate predictors of 12-month mortality post BAV } \\
\hline & OR & $95 \% \mathrm{Cl}$ & $P$ value \\
\hline $\begin{array}{l}\text { Active malignancy at } \\
\text { baseline }\end{array}$ & 4.4 & $1.3-15.1$ & 0.02 \\
\hline Smoking history & 3.3 & $1.3-7.9$ & $<0.01$ \\
\hline LVEF $\leq 30 \%$ at baseline & 3.2 & $1.3-7.6$ & $<0.01$ \\
\hline Non-elective procedure & 2.3 & $1.0-5.2$ & 0.06 \\
\hline Indication: destination & 2.2 & $1.0-4.9$ & 0.04 \\
\hline $\mathrm{BMI} \geq 30 \mathrm{~kg} / \mathrm{m}^{2}$ & 0.3 & $0.1-0.9$ & 0.03 \\
\hline
\end{tabular}

BAV, balloon aortic valvuloplasty; BMI, body mass index; LVEF, left ventricular ejection fraction; NYHA, New York Heart Association; SAVR, surgical aortic valve replacement; TAVI, transcatheter aortic valve implantation.

evaluation of their comorbidities, prognosis and psychosocial support. BAV creates an opportunity for comprehensive patient assessment to be made. Indeed, only $40 \%$ of bridging patients proceeded to TAVI or AVR within 12 months following BAV, lending support to the discriminative power and clinical utility of $\mathrm{BAV}$, as almost half of the patients originally considered for TAVI or AVR were subsequently deemed not suitable for these procedures. In this setting, BAV procedural numbers increased over the final few years of the study, mirroring the increase in TAVI procedural numbers as it becomes more frequently used among patients of lower risk.

Consistent with other studies, another important benefit of BAV is effective symptomatic relief. In our study, we have shown that in $30 \%-40 \%$ of all patients, the functional improvement (judged by the change in NYHA class of $\geq 1$ ) lasts for 6 months. Although this may seem short lived, it could provide a meaningful improvement in the quality of life in the destination group, whose life expectancy may be severely limited by other comorbidities (eg, active cancer). Our finding also has implications for the bridging group, where waitlists for TAVI remain significant in many jurisdictions. Finally, recent data suggest that emergency TAVI has high rates of 30-day mortality, and bridging BAV in this setting may provide an opportunity to medically stabilise the patient with further assessment of suitability for TAVI once stabilised. ${ }^{24}$

Immediate postprocedural haemodynamic changes were comparable with previous studies (mean gradient reduction $13.2 \pm 13.5 \mathrm{~mm} \mathrm{Hg}$ in the PARTNER Cohort B study vs $15.9 \pm 9.5 \mathrm{~mm} \mathrm{Hg}$ in our study).$^{26}$ Among our cohort, we describe low rates of major procedural complications and procedural mortality $(0.5 \%)$ also consistent with prior data, ${ }^{4619-21}$ and highlighting acceptable safety in this high-risk cohort. Twelve-month mortality was $50 \%$ among the destination treatment group, which is comparable with the $47 \%$ reported among the PARTNER Cohort B study for patients undergoing initial BAV. ${ }^{26}$ Twelve-month mortality was $26 \%$ in the bridge-to-TAVI/ AVR group, which is also similar to previously reported rates. ${ }^{16} 19$ Active malignancy at baseline, smoking history, $\mathrm{LVEF} \leq 30 \%$ at baseline and destination treatment as the indication for BAV were all associated with 12-month mortality in multivariate analysis, and therefore, careful consideration as to the likely benefit of a palliative BAV procedure in these patient groups should be made given their overall worsened prognosis.

Although numerous studies have reported BAV procedural outcomes, ${ }^{4-6} 161719$ 21-23 32-34 only one recent smaller study has assessed functional response following $\mathrm{BAV},{ }^{20}$ and none have assessed multivariate predictors of improvement in functional response post procedure. NYHA 4 at baseline, NYHA 3 at baseline, BMI $\geq 30 \mathrm{~kg} / \mathrm{m}^{2}$, female gender and bridge-to-TAVI/AVR as the indication for BAV were all associated with improvement in NYHA score at 30 days in our cohort. Our data highlight the benefit of using BAV as a bridge-to-TAVI/AVR, especially among more symptomatic patients (NYHA 3 and 4), and those of the female gender. The improved functional response and mortality benefit among obese patients has not previously been described but is consistent with the

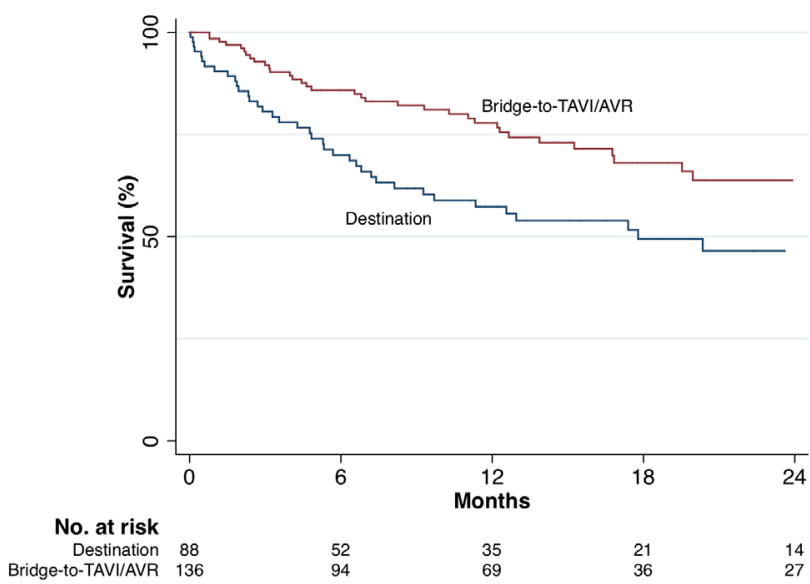

Figure 3 Kaplan-Meier survival estimates by indication - survival estimates for BAV over a 2-year follow-up. Groups compared using the log-rank test. AVR, aortic valve replacement; BAV, balloon aorticvalvuloplasty; TAVI, transcatheter aortic valve implantation. 
notion of the 'obesity paradox' described in TAVI and percutaneous coronary intervention (PCI) outcomes, whereby obesity is associated with improved post-TAVI and post-PCI survival. ${ }^{35}$ However, this observation may be at least partially driven by frail patients having lower BMI, which is not captured by our study.

Determining patients who may show improvement in LV function following BAV or TAVI/AVR is clinically important to allow the selection of patients that may maximally benefit from the procedure. Among patients with low-gradient AS, we found that $47 \%$ had improved LVEF $\geq 10 \%$ at 6 months. In our study, the only factor associated with improvement in LVEF by $\geq 10 \%$ at 6 months was LVEF $\leq 40 \%$ at baseline in our study, suggesting a higher gain in patients with more advanced disease. This may support the role of using the improvement of LV function post BAV to identify patients that may benefit most from TAVI/AVR.

Limitations of this study include its retrospective cohort design at a single metropolitan centre, which may be susceptible to selection bias and reduced generalisability to the broader Australian and international populations. As with any retrospective study, there is the potential for unmeasured confounders and findings need to be interpreted as observational. The decision regarding whether a patient underwent BAV as a destination treatment or as a bridge-to-TAVI/AVR is partially explained by the difference in STS score between groups, but generally, baseline characteristics were surprisingly similar between the groups. Frailty and cognitive function play an important role in the selection of patients for BAV and TAVI and could not be captured by this study due to the retrospective design. These factors may have assisted in explaining the difference in patient selection and outcome between the destination and bridge-to-TAVI/AVR groups.

\section{CONCLUSIONS}

BAV remains a useful clinical procedure with relatively low rates of procedural complications and mortality and has become increasingly utilised since the advent of TAVI. Contemporary indications include destination therapy, where it provides effective symptomatic relief over 6-12 months, or as a bridge-to-TAVI/AVR where it can be used as a stratifying tool for clinical decision making on valve replacement, as a measure to address issues that pose temporary contraindication to TAVI/ AVR and to offer symptomatic improvement to patients who have prolonged waiting time for the TAVI/AVR. Highly symptomatic patients (NYHA 3 or 4), females and obese patients may be more likely to benefit symptomatically following BAV.

Contributors All authors contributed to conception and design of the study. LD: performed the data collection, statistical analysis and initial manuscript drafting. LD and SD: responsible for the overall content. All authors contributed to conception and design of the study; contributed to interpretation of results and critical revision of the manuscript.
Funding Professor Duffy's work is funded by National Health and Medical Research Council grants.

Competing interests None declared.

Patient consent for publication Not required.

Provenance and peer review Not commissioned; externally peer reviewed.

Data availability statement Data are available upon reasonable request. Data are available upon reasonable request.

Open access This is an open access article distributed in accordance with the Creative Commons Attribution Non Commercial (CC BY-NC 4.0) license, which permits others to distribute, remix, adapt, build upon this work non-commercially, and license their derivative works on different terms, provided the original work is properly cited, appropriate credit is given, any changes made indicated, and the use is non-commercial. See: http://creativecommons.org/licenses/by-nc/4.0/.

ORCID iDs

Luke Dawson http://orcid.org/0000-0003-3789-5808

Dion Stub http://orcid.org/0000-0001-8686-2709

\section{REFERENCES}

1 Eggebrecht $\mathrm{H}$, Mehta $\mathrm{RH}$. Transcatheter aortic valve implantation (TAVI) in Germany 2008-2014: on its way to standard therapy for aortic valve stenosis in the elderly? Eurolntervention 2016;11:1029-33.

2 Grover FL, Vemulapalli S, Carroll JD, et al. 2016 Annual Report of The Society of Thoracic Surgeons/American College of Cardiology Transcatheter Valve Therapy Registry. J Am Coll Cardiol 2017;69:1215-30.

3 Meredith IT, Walton A, Walters DL, et al. Mid-Term outcomes in patients following transcatheter aortic valve implantation in the CoreValve Australia and New Zealand study. Heart Lung Circ 2015;24:281-90.

4 Alkhouli M, Zack CJ, Sarraf M, et al. Morbidity and mortality associated with balloon aortic valvuloplasty: a national perspective. Circ Cardiovasc Interv 2017;10.

5 Badheka AO, Patel NJ, Singh V, et al. Percutaneous aortic balloon valvotomy in the United States: a 13-year perspective. Am J Med 2014;127:744-53.

6 Saia F, Marrozzini C, Ciuca C, et al. Emerging indications, inhospital and long-term outcome of balloon aortic valvuloplasty in the transcatheter aortic valve implantation era. Eurolntervention 2013;8:1388-97.

7 Jabbour RJ, Stub D, Walton A. Aortic valvuloplasty - is a revival merited? Br J Cardiol 2011;18:34-6.

8 Moreno PR, Jang IK, Newell JB, et al. The role of percutaneous aortic balloon valvuloplasty in patients with cardiogenic shock and critical aortic stenosis. J Am Coll Cardiol 1994;23:1071-5.

9 Cribier A, Savin T, Saoudi N, et al. Percutaneous transluminal valvuloplasty of acquired aortic stenosis in elderly patients: an alternative to valve replacement? Lancet 1986;1:63-7.

10 Berland J, Cribier A, Savin T, et al. Percutaneous balloon valvuloplasty in patients with severe aortic stenosis and low ejection fraction. immediate results and 1-year follow-up. Circulation 1989;79:1189-96.

11 Lieberman EB, Bashore TM, Hermiller JB, et al. Balloon aortic valvuloplasty in adults: failure of procedure to improve long-term survival. J Am Coll Cardiol 1995;26:1522-8.

12 Otto CM, Mickel MC, Kennedy JW, et al. Three-Year outcome after balloon aortic valvuloplasty. Insights into prognosis of valvular aortic stenosis. Circulation 1994;89:642-50.

13 Theiss HD, Greif M, Steinbeck G, et al. Balloon valvuloplasty for treatment of cardiogenic shock in the era of surgical valve replacement and TAVI. Intern Emerg Med 2014;9:345-7.

14 Kogoj P, Devjak R, Bunc M. Balloon aortic valvuloplasty (BAV) as a bridge to aortic valve replacement in cancer patients who require urgent non-cardiac surgery. Radiol Oncol 2014;48:62-6.

15 Safian RD, Berman AD, Diver DJ, et al. Balloon aortic valvuloplasty in 170 consecutive patients. N Engl J Med 1988;319:125-30.

16 Ben-Dor I, Maluenda G, Dvir D, et al. Balloon aortic valvuloplasty for severe aortic stenosis as a bridge to transcatheter/surgical aortic valve replacement. Catheter Cardiovasc Interv 2013;82:632-7.

17 Araque JC, Greason KL, Suri RM, et al. The Role of Balloon Aortic Valvuloplasty in Patients With Aortic Valve Stenosis and Society of Thoracic Surgeons Risk of $15 \%$ or Higher. Ann Thorac Surg 2016;101:592-8. 
18 Dawkins S, Makkar RR. Balloon aortic valvuloplasty: is it still relevant? Circ 2017;10.

19 Moretti C, Chandran S, Vervueren P-L, et al. Outcomes of patients undergoing balloon aortic valvuloplasty in the TAVI era: a multicenter registry. J Invasive Cardiol 2015;27:547-53.

20 Daniec M, Nawrotek B, Sorysz D, et al. Acute and long-term outcomes of percutaneous balloon aortic valvuloplasty for the treatment of severe aortic stenosis. Catheter Cardiovasc Interv 2017:90:303-10.

21 Eltchaninoff H, Durand E, Borz B, et al. Balloon aortic valvuloplasty in the era of transcatheter aortic valve replacement: acute and longterm outcomes. Am Heart J 2014;167:235-40.

22 Malkin CJ, Judd J, Chew DP, et al. Balloon aortic valvuloplasty to bridge and triage patients in the era of trans-catheter aortic valve implantation. Catheter Cardiovasc Interv 2013;81:358-63.

23 Saia F, Moretti C, Dall'Ara G, et al. Balloon aortic valvuloplasty as a bridge-to-decision in high risk patients with aortic stenosis: a new paradigm for the heart team decision making. J Geriatr Cardiol 2016;13:475-82.

24 Bongiovanni D, Kühl C, Bleiziffer S, et al. Emergency treatment of decompensated aortic stenosis. Heart 2018;104:23-9.

25 Wijeysundera HC, Wong WWL, Bennell MC, et al. Impact of wait times on the effectiveness of transcatheter aortic valve replacement in severe aortic valve disease: a discrete event simulation model. Can J Cardiol 2014;30:1162-9.

26 Kapadia S, Stewart WJ, Anderson WN, et al. Outcomes of inoperable symptomatic aortic stenosis patients not undergoing aortic valve replacement: insight into the impact of balloon aortic valvuloplasty from the partner trial (placement of aortic transcatheter valve trial). JACC Cardiovasc Interv 2015;8:324-33.

27 Cutlip DE, Windecker S, Mehran R, et al. Clinical end points in coronary stent trials: a case for standardized definitions. Circulation 2007;115:2344-51.
28 Mehran R, Rao SV, Bhatt DL, et al. Standardized bleeding definitions for cardiovascular clinical trials: a consensus report from the bleeding academic research Consortium. Circulation 2011;123:2736-47.

29 Outcomes (KDIGO) Acute KidneyInjury Work Group KI. KDIGO clinical practice guidelines for acute kidney injury. Kidney Int Suppl 2012;2:1-138.

30 Nishimura RA, Otto CM, Bonow RO, et al. 2017 AHA/ACC Focused Update of the 2014 AHA/ACC Guideline for the Management of Patients With Valvular Heart Disease: A Report of the American College of Cardiology/American Heart Association Task Force on Clinical Practice Guidelines. J Am Coll Cardiol 2017;70:252-89.

31 Nishimura RA, Otto CM, Bonow RO, et al. 2014 AHA/ACC guideline for the management of patients with valvular heart disease: a report of the American College of Cardiology/American Heart Association Task Force on Practice Guidelines. [Erratum appears in J Am Coll Cardiol. 2014 Jun 10;63(22):2489. Dosage error in article text]. J Am Coll Cardiol 2014;63:e57-185.

32 Christodoulidis G, Yu J, Kini A, et al. Gender-Specific outcomes after balloon aortic valvuloplasty: inhospital and long-term outcomes. Am Heart J 2015;170:180-6.

33 Jabbour RJ, Dick R, Walton AS. Aortic balloon valvuloplasty--review and case series. Heart Lung Circ 2008;17 Suppl 4:S73-81.

34 Khawaja MZ, Sohal M, Valli H, et al. Standalone balloon aortic valvuloplasty: indications and outcomes from the UK in the transcatheter valve era. Catheter Cardiovasc Interv 2013;81:366-73.

35 Konigstein M, Havakuk O, Arbel Y, et al. The obesity paradox in patients undergoing transcatheter aortic valve implantation. Clin Cardiol 2015;38:76-81.

36 Lancefield T, Clark DJ, Andrianopoulos N, et al. Is there an obesity paradox after percutaneous coronary intervention in the contemporary era? an analysis from a multicenter Australian registry. JACC Cardiovasc Interv 2010;3:660-8. 